Available online on 15.09 .2017 at http://jddtonline.info
Journal of Drug Delivery and Therapeutics
Open Access to Pharmaceutical and Medical Research
$\begin{gathered}\text { O 2011-17, publisher and licensee JDDT, This is an Open Access article which permits unrestricted non- } \\ \text { commercial use, provided the original work is properly cited }\end{gathered}$

Open

Access

Research Article

\title{
COMPARISON OF NECK ISOMETRICS VS A COMBINATION OF NECK ISOMETRICS AND SHOULDER ISOMETRICS IN RELIEVING NECK PAIN AND IMPROVING STRENGTH
}

\author{
Chhavi Singh ${ }^{1}$, Harsh Omer ${ }^{2}$ \\ ${ }^{1}$ BPT, MPT, CMT, MIAP, Assistant Professor, Faculty of Physiotherapy and Diagnostics, Jayoti Vidhyapeeth Women's University, Jaipur \\ (RJ.), India \\ ${ }^{2}$ BPT, M.Sc YOGA, CMT, MIAP, Dip. In Osteopath (Canada), Clinical Therapist, Jayoti Vidhyapeeth Women's University, Jaipur (RJ.), \\ India
}

\begin{abstract}
Introduction: Neck disorders remain a common problem in modern world. Patients with chronic neck pain uses health care services twice as much as the population on average. The origin of neck pain can be multifactorial. Aim and objectives: To determine whether only neck muscle isometrics or neck along with shoulder muscle isometrics are more effective in relieving chronic neck pain. Methodology: 30 female subjects with mean age $20.23 \pm 1.96$ yrs, have been included in the study as the sample size with 10 equal subjects assigned to each group with mean age of $20.5 \pm 2.20$ in group A, 19.8 \pm 1.37 in group B, 20.4 \pm 2.24 in group C as per inclusion criteria via random sampling method. Conclusion: Results of the study shows that pain \& strength of neck muscles were improved in the subjects with neck pain individually in all the groups but does not show any significant improvement among the groups. Thus, neck pain can be reduced after training periods of neck muscles and shoulder muscles with intensive strength.
\end{abstract}

Key words: VAS, Muscle strength, Strain Guage, Thera Band.

Article Info: Received 10 July, 2017; Review Completed 03 Sep, 2017; Accepted 04 Sep, 2017; Available online 15 Sep, 2017

Cite this article as:

Singh S, Omer H, Comparison of neck isometrics Vs a combination of neck isometrics and shoulder isometrics in relieving neck pain and improving strength, Journal of Drug Delivery and Therapeutics. 2017; 7(5):80-85

DOI: http://dx.doi.org/10.22270/jddt.v7i5.1490

*Address for Correspondence

Chhavi Singh. BPT, MPT, CMT, MIAP, Assistant Professor, Faculty of Physiotherapy and Diagnostics, Jayoti Vidhyapeeth Women's University, Jaipur (RJ.), India

\section{INTRODUCTION}

Neck disorders remain a common problem in modern world. Neck pain has been the most common chief complaint among the college going females. Mild neck pain is experienced by nearly everyone at sometime or the other in their lives. In general population, neck pain and dysfunction are common, affecting up to $67 \%$ of general population at some time during their life. It is also often accompanied by headache and arm pain. Painful conditions appear in most cases as a result of excessive mental or physical stress. Patients with chronic neck pain used health care services twice as much as the population on average.
The exact origin and pathophysiologic mechanisms of chronic neck pain often remains obscure because trauma or severe degenerative conditions at working age are found only in a few cases. The origion of neck pain can be multifactorial. Excessive physical strain may cause micro trauma in connective tissues. Degenerative changes in cervical vertebra and discs, muscles, ligaments, zygapophyseal joints, dura or nerve root are common and increases with advanced age in asymptomatic people ${ }^{1}$. Even the causes of neck pain can be frozen shoulder, thoracic outlet syndrome, scoliosis, nutritional deficiencies, fibromyalgia, poor posture. Such "non specific" neck problems are costly in terms of disability and work loss ${ }^{3}$. 
Various other factors responsible for neck pain are like neck muscle strength, neck muscle endurance. Peak isometric neck strength values are significantly reduced in women with chronic neck pain compared with healthy controls in all the directions tested. A positive relation has been found between various neck disorders and work related risk factors such as static neck and arm posture, duration of sitting and work place design, high quantitative job demands and limited rest break opportunities have been found as predictor of neck pain. Among individual role of psychological factors has been emphasized. Insufficient muscular strength can contribute to major functional losses, of even the most basic activities of daily living 5 .

Only a few studies have focused on the strength of neck muscles in patients with non specific, chronic neck pain. Neck pain is often accompanied by shoulder and arm pain and majority of pain that travels down to shoulder and arm from neck is - referred pain. Shoulder is usually not the problem. It may feel that way but actually shoulder joint will not be at fault. In fact, the first 5-10 degrees of movement in shoulder comes from shoulder joint and rest relies on upper back and neck, the shoulder blade and collar bones. If these areas work incorrectly then shoulder pain occurs. Reason to involve shoulder muscle isometrics was that expressions of pain in lower fibers of trapezious muscle are also found in patients with chronic neck pain. While actively employed, women experience sensations of tenderness on touch, stiffness, constant muscle fatigue and more especially pain from trapezious muscle.

Different prevention and treatment techniques being tried to cure neck pain are exercise therapy for strengthening, electrotherapy to cure pain, yoga, fibromyalgic diet, moist heat, hard styrofoam rollers, trigger point therapy, ergonomic advisors, sick leave therapy etc. The symptoms usually disappear quickly with only a few individuals, actually consulting a doctor.

Despite of its high incidence, neck pain is poorly understood. Neck pain arising from cervical spine is typically precipitated by movements and may be accompanied by focal tenderness and limitation of movements, yet neck muscle functioning is usually accepted as satisfactory, if the gross movements of the neck are normal. Many studies have investigated the relationship between neck pain and working conditions. Students population is much affected by neck pain disorders, due to the continues studies for longer duration in a particular posture i.e. basically in flexed neck position. The relation between physical load at work and neck pain may be influenced by the level of physical fitness of the students.

Statement of the study:

Whether only neck muscle isometrics or neck along with shoulder muscle isometrics are more effective in relieving chronic neck pain?

Aim and purpose of study:

In this study, we revealed that this method is a non invasive and cost effective method which provides more generalization of norms and also assures to be a user friendly. We did the comparison of the effect of only neck muscle isometrics with neck and shoulder muscle isometrics in relieving pain. These treatment protocols can be useful in clinical settings to establish prognostic criteria of the patients as it can address unhelpful beliefs that may contribute to development, maintenance of disability by catering to the psychosocial needs of the patient and thus help in proper post rehabilitation assessment.

\section{METHODOLOGY:}

This study is a comparative study design in which 30 subjects were taken by random sampling method i.e, 30 female hostellers of Mata Gujri hostel, Dehradun was taken._Inclusion Criteria is females of age group 18 to 25 years, regular college going females, constant or frequently occurring neck pain for more than 6 months, no history of any recent injury to neck. The exclusion criteria is cervical spine disorders such as disc prolapsed, Spinal stenosis, post operative conditions in neck and shoulder area, history of severe trauma, Frequent migrane, Severe psychiatric conditions.

\section{Procedure:}

30 normal females with mean age of $20.23 \pm 1.96$ years were assessed and selected according to selection criteria after the ethical committee approval. All the participants were given verbal instructions for the testing procedure \& informed consent form was obtained from each one of them, prior to the participation in the study.

The subjects were screened so as to fulfill the inclusion criteria (refer appendix-D). After that neck muscle strength for flexion, extension, right lateral flexion and left lateral flexion \& right and left shoulder muscle strength for flexion, extension, abduction and adduction was measured at 0 session. VAS was also marked. Three readings were taken and their mean was taken for accuracy. Next random sampling of the subjects was done to divide them into 4 equal groups i.e., group $\mathrm{A}$ $(\mathrm{n}=10)$, group $\mathrm{B}(\mathrm{n}=10)$, group $\mathrm{C}(10)$ with mean age of $20.5 \pm 2.20,19.8 \pm 1.37,20.4 \pm 2.24$ in respective groups. Each subject was then treated to her respective group.

The subjects were motivated to do their best. Warm up was given prior to each session.

In group $\mathrm{A}$, hot pack for 10 minutes was given. Then subjects were asked to voluntarily do neck muscle isometrics in a set of 10 repetitions with 5 seconds hold and 5 seconds rest in all four directions. A sustained stretch to used muscles was given to avoid doms.

For group B, again hot pack was given for 10 minutes. Then subjects were asked to voluntarily do neck and shoulder muscle isometrics in a set of 10 repetitions with 5 seconds hold and 5 seconds rest in all four directions. Sustained stretch to used muscles of neck and shoulder muscles was given.

For group C, only hot pack for 10 minutes was given. After strengthening was over, again VAS and STRENGTH was measured. In each group 3 readings were taken and then mean was evaluated for accuracy. 
This strengthening technique was continued for 9 sessions and measured at the end of $9^{\text {th }}$ session.

Data Analysis: One way ANOVA has been performed for comparing the pre treatment VAS \& STRENGTH at 0 week and post treatment at $3^{\text {rd }}$ week, between groups A, B, C. It tells whether there are general, non-specified differences in the results from the different conditions.

\section{RESULT:}

In this study, 30 subjects with mean age of 20.2334 1.96 yrs were taken, 3 groups were made i.e., A, B, C and 10 subjects were included in each group. The mean age of subjects in group A, B, C were taken as $20.5 \pm$ $2.202,19.8 \pm 1.372,20.4 \pm 2.245$. (Refer to table 1.1)

Table 1: Subject Information

\begin{tabular}{|c|c|c|c|}
\hline Sr. no. & Groups & no. of subjects & Mean age \pm S.D \\
\hline 1 & A & 10 & $20.5 \pm 2.202$ \\
\hline 2 & B & 10 & $19.8 \pm 1.372$ \\
\hline 3 & C & 10 & $20.4 \pm 2.245$ \\
\hline
\end{tabular}

Comparison of mean vas and strength of neck pre exercise between groups $\mathrm{A}, \mathrm{B}, \mathrm{C}$.

One way ANOVA has been performed for 0 session VAS \& neck muscle strength measurement. The result shows non- significant difference $(\mathrm{p}>0.05)$ and the mean of group A, B, C for anova were evaluated. The variables were VAS, neck flexion, neck extension, neck right lateral flexion, neck left lateral flexion for which values were $7.6 \pm 1.265,3.74 \pm 1.180,5.98 \pm 1.929,4.5$ $\pm 1.1333,4.2 \pm 1.070 ; 7.8 \pm 1.317,3.7 \pm 1.660,5.19 \pm$ $1.808,3.47 \pm 1.142,3.79 \pm 0.807 ; 5.8 \pm 1.135,3.71 \pm$ $0.675, \quad 5.15 \pm 0.704,3.97 \pm 0.702,3.83 \pm 0.831$ for group A, B, C respectively.(Refer to table 1.2)

Comparison of mean strength of right shoulder strength pre exercise between group $\mathrm{A}, \mathrm{B}, \mathrm{C}$.

One way ANOVA has been performed for 0 session right shoulder strength measurement. The result shows non- significant difference $(p>0.05)$ and the mean of group A, B, C for anova were evaluated. The variables were flexion, extension, abduction and adduction for which values were $3.74 \pm 1.184,4.52 \pm 1.064,4.75 \pm$ $1.826,4.55 \pm 1.850 ; 4.02 \pm 0.520,4.53 \pm 0.609,4.98 \pm$ $1.338,4.42 \pm 1.257 ; 3.52 \pm 0.857,4.37 \pm 0.820,4.99 \pm$ $1.649,3.88 \pm 1.160$ for group A, B, C respectively. (Refer to table 1.2)

Comparison of mean strength of left shoulder strength pre exercise between group $\mathrm{A}, \mathrm{B}, \mathrm{C}$.

One way ANOVA has been performed for 0 session left shoulder strength measurement. The result shows nonsignificant difference $(p>0.05)$ and the mean of group A, $\mathrm{B}, \mathrm{C}$ for anova were evaluated. The variables were flexion, extension, abduction and adduction for which values were $3.88 \pm 1.655,3.99 \pm 1.048,5.36 \pm 1.918$, $4.2 \pm 1.2 ; 3.7 \pm 0.752,3.97 \pm 0.684,6 \pm 1.794,4.16 \pm$ $0.932 ; 3.75 \pm 1.017,4.05 \pm 0.774,5.32 \pm 1.307,3.51 \pm$ 0.589 for group A, B, C respectively. (Refer table 1.2)

Table 2: comparison of pre vas and mean strength for group A, B \& C

\begin{tabular}{|c|c|c|c|c|c|c|c|c|c|}
\hline & $v_{1}$ & $\mathrm{MF}$ & $\mathrm{ME}$ & NRLF & XLLF & RTSHE & RT SHE & RT SH A9D & RT SHACO \\
\hline A & $7 . t e 1264$ & $3.74 \pm 1.264$ & $593 \pm 1929$ & $42 \pm 10708$ & $4.5 \pm 1.1333$ & $3.74 \pm 1.1843$ & $452 \pm 10643$ & $475 \pm 1.8265$ & $455=1.350075$ \\
\hline B & 7.t.ta1.3165 & $3.7 \pm 1.9060$ & $519+1929$ & $3.79 \pm \pm: 8075$ & $3.47 \pm 1.1421$ & $4.02+0.520$ & $453 \pm 0629$ & $495+13385$ & $4.42 \div 12575$ \\
\hline c & $5.8 x+1.135$ & $3.71 \pm 2.675$ & $515+070 \times 3$ & $3.83+0.8313$ & $3.97 \pm 2.7024$ & $3.52+0.257$ & $437 \pm 0.0206$ & $499+16496$ & $3.89=1.1622$ \\
\hline Frtaus & 16 & 2567 & 0.8778 & 0.615 & 2562 & 0.722 & 0.11 & 000 & 0.596 \\
\hline Signifcency & 5 & N.S & NS & NS & N.S & NS & NS & NS & N.S \\
\hline
\end{tabular}

Comparison of mean vas and strength of neck post exercise between group $\mathrm{A}, \mathrm{B}, \mathrm{C}$

One way ANOVA has been performed for $9^{\text {th }}$ session VAS \& neck muscle strength measurement. The result shows non- significant difference $(\mathrm{p}>0.05)$ and the mean of group A, B, C for anova were evaluated. The variables were VAS, neck flexion, neck extension, neck right lateral flexion, neck left lateral flexion for which values were $3.1 \pm 2.131,4.26 \pm 0.822,7.05 \pm 1.450$ $5.41 \pm 1.299,5.26 \pm 1.444 ; 3.2 \pm 1.135,4.37 \pm 1.373$, $6.08 \pm 2.106,4.37 \pm 1.082,4.62 \pm 1.561 ; 2.2 \pm 1.135$, $4.38 \pm 0.415,5.89 \pm 1.321,4.21 \pm 0.795,4.24 \pm 0.768$ for group A, B, C respectively. (Refer to table 1.3). 
Comparison of mean strength of right shoulder strength post exercise between group $\mathrm{A}, \mathrm{B}, \mathrm{C}$.

One way ANOVA has been performed for $9^{\text {th }}$ session right shoulder strength measurement. The result shows non- significant difference $(p>0.05)$ and the mean of group A, B, C for anova were evaluated. The variables were flexion, extension, abduction and adduction for which values were $3.89 \pm 0.868,4.63 \pm 0.801,5.21 \pm$ $1.457,4.6 \pm 0.980 ; 3.83 \pm 0.614,4.41 \pm 0.672,5.36 \pm$ $0.975,4.47 \pm 1.204 ; 3.83 \pm 0.651,4.14 \pm 0.658,5.02 \pm$ $1.084,3.61 \pm 0.715$ for group $\mathrm{A}, \mathrm{B}, \mathrm{C}$ respectively. (Refer to table 1.3)
Comparison of mean strength of left shoulder strength post exercise between group $A, B, C$.

One way ANOVA has been performed for $9^{\text {th }}$ session left shoulder strength measurement. The result shows non- significant difference $(p>0.05)$ and the mean of group A, B, C for anova were evaluated. The variables were flexion, extension, abduction and adduction for which values were $4.06 \pm 1.081,4.37 \pm 0.689,4.95 \pm$ $1.444,4.28 \pm 0.961 ; 3.79 \pm 0.724,4.24 \pm 0.839,5.26 \pm$ $0.767,3.93 \pm 1.006 ; 3.84 \pm 0.965,4.35 \pm 0.824,5.28 \pm$ $0.972,3.91 \pm 0.768$ for group $\mathrm{A}, \mathrm{B}, \mathrm{C}$ respectively. (Refer table to table 1.3).

Table 3: comparison of post vas and mean strength for group A, B \& C

\begin{tabular}{|c|c|c|c|c|c|c|c|c|c|c|c|}
\hline & n2 & NF2 & NE2 & NRLF2 & NLLF2 & RT $=2$ & RTE2 & RTA302 & RTADD2 & LTF2 & LTE2 \\
\hline A & 3.1.12:131 & 42600.822 & $7.6 \pm 11450$ & $5.41 \pm 1.280$ & $526 \pm 1.445$ & $389-186805$ & $4.63 \pm 0.00145$ & $521 \pm 1.4578$ & $4.6 \pm 0.961$ & $4.05 \pm^{1} 1061$ & $4.37+0.689$ \\
\hline B & $32+1.135$ & $4.37 \pm 1.373$ & $6.08+21064$ & $4.37 \pm 1.62$ & $4.62+1.561$ & 38000615 & $4.41 \pm 0.672$ & $5.36 \pm 0.975$ & $4,47 \pm 1205$ & $379+0.76$ & $424+0.839$ \\
\hline C & $22+1.135$ & $\angle 38+0.416$ & $580 \pm 1321$ & $4.21 \pm 0.786$ & $4.24+0.706$ & $3 R=0662$ & $4.14 \pm 0060$ & $502+1.084$ & $3.61 \pm 0776$ & $3.84 \pm 0.965$ & $4,35+1,825$ \\
\hline Fia.e & $2.340^{\circ}$ & $00480^{-1}$ & 1.4008 & 3.650 & 155950 & 0.02312 & 1.8624 & 02048 & 2.5679 & 0.8055 & 0.0789 \\
\hline Significacy & NS & NS & NS & S & NS & NS & NS & NS & N.S & NS & NS \\
\hline
\end{tabular}

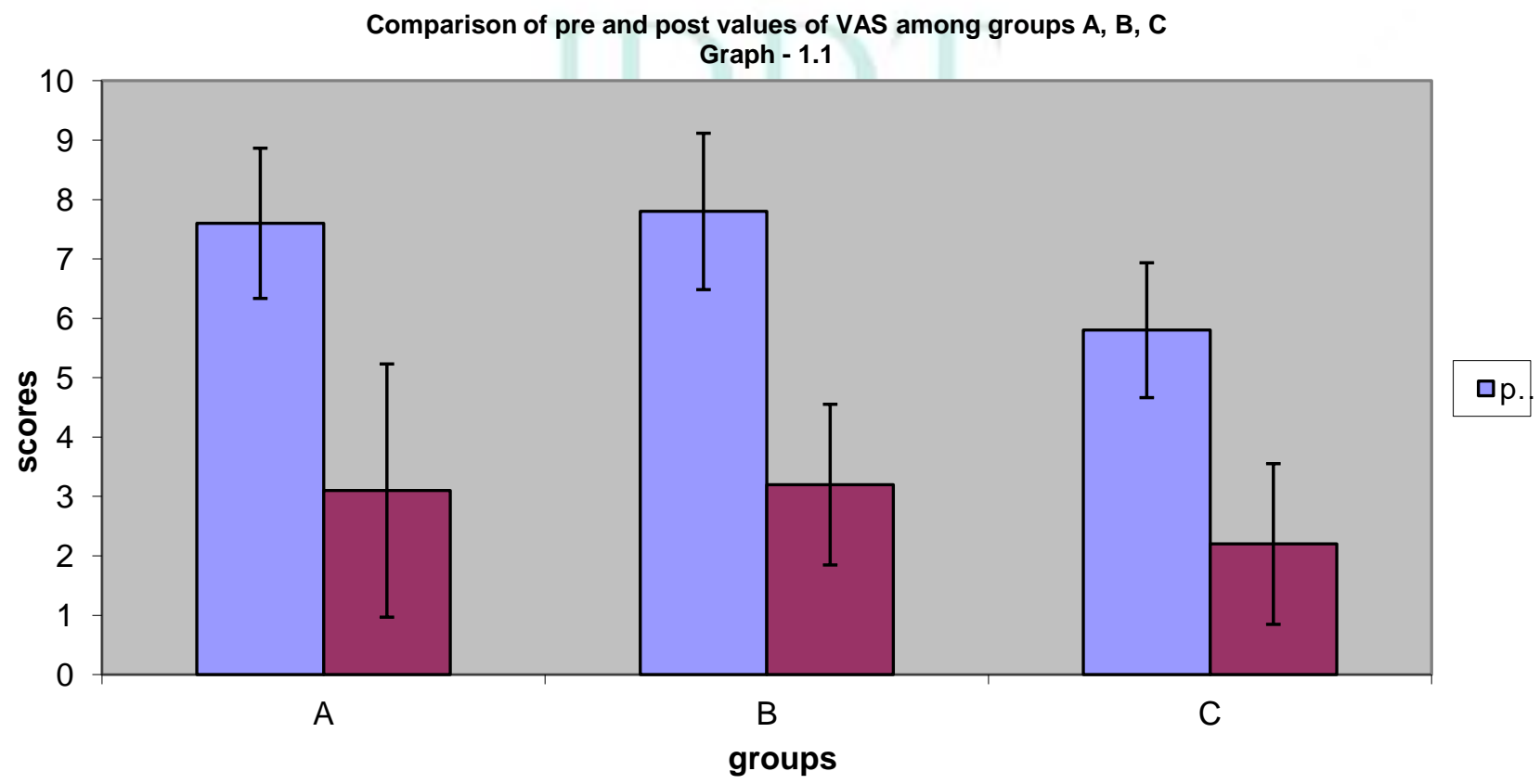


Comparision of pre and post values of different movement strength in neck among 3 groups.

Graph - 1.2
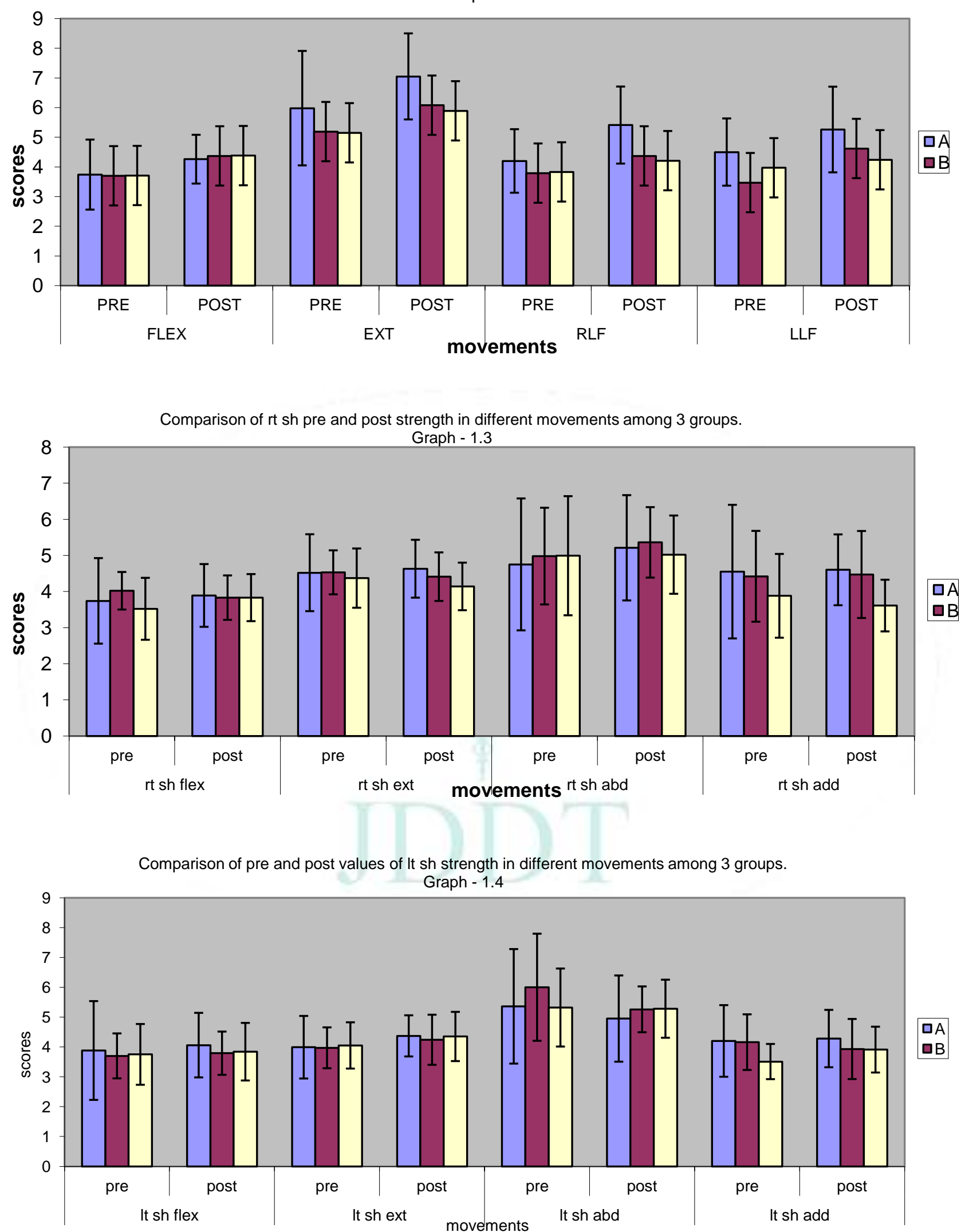

\section{DISCUSSION}

In this study a comparison has been made between the effect of isometric neck muscle strengthening and a combination of the neck and shoulder muscle isometrics, in the subjects with neck pain. No standard method for the treatment of non traumatic neck pain has yet been established. In this study, an initial assessment was done for the neck and shoulder muscle strength as well as pain scores for the neck pain subjects. The measurement protocol was designed to study the changes in pain scores, through VAS, and neck and shoulder muscles 
strength after isometric strengthening, for 9 sessions. One way anova was used for the data analysis between the two readings. As per the results, no significant difference was found in pain scores and strength of both neck and shoulder muscles among the groups.

Strengthening exercises are done in order to keep the neck in proper position to prevent impingement, improve functional capacity, preventing and recovering from injuries and maintaining length tension relationship of the musculature.

Cristopher M Narres, (1999) muscular contraction involves a combination of physiological and neurological processes and consequently adaptations to resistance training are both myogenic (structural) and neurogenic (seen on EMG only) in nature.

Astrand and Rodahl, (1986) significant neurogenic gains may be made at the beginning of the strength training programme without noticeable changes in myogenic strength.

G.S Loi and L. Landon (1989) concluded it is possible to obtain an increase in strength without an adaptation in muscle fibre but not without an adaptation in nervous system. Joel A. Delisa et al (2005) according to them neural adaptations due to resistance training are responsible for increase in strength that proceeds and increase in muscle size during early phase of resistance training program. Kisner (2002) found strength gain observed early in resistance training program (after 3 to 4 weeks) is the result of neural adaptations. For significant changes to occur in muscle, such as hypertrophy or increased vascularisation at least 6 to 12 weeks were required.

\section{REFERENCES}

1. Bogduk et al: 1998: Neck pain: an update: Aust Farm Physician: 17: 75-86

2. International association for the study of pain: 1779: Pain Terms: A list with definition and notes on usage. Pain 6, 24952.

3. Aker P D et al: 1996: British med jour 313: Conservative management of mechanical neck pain: systemic overview and meta- analysis: 1291-1296.

4. Caroyln Kisner and Lynn Allen Colby: 2003: Therapeutic Exercise: Resistance exercise: 1:58-148.

5. Caroyln Kisner and Lynn Allen Colby : Therapeutic exercises foundation and technique: $4^{\text {th }}$ edition: chapter $3^{\text {rd }}$ : page 60 .

6. Tezel Aet al: 2005 feb:Musculoskeletal disorders in left and right handed dental students.International Journal Narosci. 115(2):225-66

7. Carlson A M: 1983: Assessment of chronic pain: aspect of reliability and validity of VAS pain 16:87.

8. Edward A Shipton: 1999: Pain acute and chronic, $2^{\text {nd }}$ edition, Arnold and oxford University Press 1-33.

9. G A M Aries et al: 2001: march: Are neck flexion, neck rotation and sitting at work risk factor for neck pain? Results of a prospective cohort study: occup environ med: 58:200207.
Leuithi et al (1986) found an increase of $8.4 \%$ in cross sectional area of muscles following a 6 week period of resistance exercise. Groups training between 3 and 5 days per week recorded significantly greater strength increases than those subjects who exercised only one day per week. Training 3 days and 5 days per week was found to be superior to training 2 days per week. Although the mean strength increases of group training 3 days per week was substantially greater than the group training 2 days per week, no statistical differences was established. No significantly differences in strength acquisition were observed between training 1 day per week and neither 2 days per week nor 3 days per week and 4 days per week. Henderson found that weight training 3 days per week for one hour per day was significantly better than 2 days per week for one and one-half hour per day for increasing muscular strength ${ }^{46}$. Newman - keuls procedure indicated that improvement of muscular strength was significantly greater in the group that trained 5 days per week than groups training fewer frequencies per week. The results of study "effects of frequency of weight training on muscle strength enhancement" by G. McKENZIE GILLAM appear to contradict the commonly practiced principle that maximum muscular strength improvement might be obtained through weight training frequencies of less than 5 days per week. Participating in physical education classes 5 days per week has been observed to superior to 3 and 2 days per week for improving physical fitness, strength and skill acquisition ${ }^{487}$. Collectively these findings appear to imply that more frequent the stress the greater the adaptation.
10. Talat Naheed et al: 2006: Neck pain- A problem with major effects. Pak J. med science, vol.22,no.2, 188-190.

11. Holly A Sweeney: Avoiding repetitive stress injuries how the Alexander technique can help.

12. Carol A Oatis: 2004, Mechanics and pathomechanics of spine, kinesiology. The mechanics and pathomechanics of human movement: Lippincott William and Wilkins, $3^{\text {rd }}$ edition, page no. 473-486.

13. Louis Salmon: Apley's system of orthopadics and fracture management, $8^{\text {th }}$ edition, page $357-359,367$.

14. Gosselin G et al: 2004: June:Effect of neck extensors muscle fatigue on balance: Clinical Biomech: 19(5): 473-9.

15. Burgers- lime rick et al: The influences of computer monitor height on head and neck posture. International Journal of Industrial Ergonomics Source: SHN report.

16. Seng K Y et al: 2002: August: Neck muscle strength across sagittal and coronal plane: an isometrics study: clinical biomech: 17(7):545-7.

17. Treiber F. A., Lott J, Dunchan J., Slavens G., Davis H.,1998: July- August: Effect of thera band and light weighted dumbbell training on shoulder rotator torque and serve performance inb college tennis players: AM J sports med: 26(4): 510-15. 